\title{
Desenvolvimento de licores de fruta nativa Curriola (Pouteria ramiflora), avaliação proximal e aceitabilidade
}

\author{
Development of liqueurs of Curriola (Pouteria ramiflora) native fruit, proximal evaluation and \\ acceptability
}

Desarrollo de licores de frutas nativas Curriola (Pouteria ramiflora), evaluación proximal y aceptabilidad

Geriel Araujo Lemes

ORCID: https://orcid.org/0000-0002-1386-6861 Universidade Federal de Mato Grosso, Brasil E-mail: geriellemes@gmail.com

Maria da Graça Tomás

ORCID: https://orcid.org/0000-0003-4106-9787 Universidade Federal de Mato Grosso, Brasil E-mail: mdagraca.tomas@gmail.com

Adelino da Cunha Neto

ORCID: https://orcid.org/0000-0003-4365-9502 Universidade Federal de Mato Grosso, Brasil

E-mail: adeneto@yahoo.com.br

Maressa Caldeira Morzelle

ORCID: https://orcid.org/0000-0001-8841-6630 Universidade Federal de Mato Grosso, Brasil E-mail: maressamorzelle@hotmail.com

Priscila Becker Siqueira

ORCID: https://orcid.org/0000-0001-6282-3991 Universidade Federal de Mato Grosso, Brasil E-mail: pribecker@gmail.com

Luiz José Rodrigues

ORCID: https://orcid.org/0000-0002-1486-1843 Universidade Federal de Mato Grosso, Brasil E-mail: rodrigues.luizjose78@gmail.com José Masson

ORCID: https://orcid.org/0000-0003-3133-5471 Instituto Federal de Educação, Ciência e Tecnologia de Mato Grosso, Brasil E-mail:jose.masson@blv.ifmt.edu.br

\begin{abstract}
Resumo
Este estudo objetivou caracterizar a composição centesimal do fruto da currioleira (Pouteria ramiflora) e verificar características físico-química e sensoriais de licores do fruto. Quantificou-se teores de umidade, fibra alimentar, proteína, lipídeos, cinzas e carboidratos e o valor energético da polpa do fruto. Preparou-se licores da polpa e fruto inteiro, com concentrações de açúcar de 150 e de $250 \mathrm{~g} . \mathrm{L}^{-1}$. Os licores foram submetidos a análise sensorial de aceitação e intenção de compra e determinados o teor alcoólico, acidez total, $\mathrm{pH}$, sólidos solúveis, parâmetros de cor, compostos fenólicos totais e capacidade antioxidante. Na composição centesimal da polpa de curriola destaca-se o alto teor de fibras $12,75 \mathrm{~g} \cdot 100 \mathrm{~g}^{-1}$ e baixa caloria $54,11 \mathrm{kcal}^{-100 \mathrm{~g}^{-1}}$. Diferenças significativas $\mathrm{p}<0,05$ foram observadas nos licores nas análises físico-químicas e sensoriais. Licores de polpa obtiveram maior intensidade de amarelo com médias de $b^{*} 44,36$ e 44,42 e tenderam a coloração verde com valores negativos de $a^{*}-0,89$ e -1,87, em contrapartida licores de fruto integral apresentaram maior grau de vermelho com valores positivos de a* 9,6 e 5,43. A cor de licores de polpa foi menos aceita $(4,23$ e 4,32) em relação aos licores do fruto integral $(5,46$ e 5,46$)$. As concentrações alcoólicas de 20,58, 22,75, 33,58 e 42,75 influenciaram na impressão global, no sabor e na intenção de compra dos licores. Os licores do fruto curriola com menores teores alcoólicos foram os que obtiveram a melhor aceitação.
\end{abstract}

Palavras-chave: Pouteria ramiflora; Composição proximal; Licor; Aceitabilidade; Bebida alcoólica.

\section{Abstract}

This study aimed to characterize the centesimal composition of the fruit of the currioleira (Pouteria ramiflora) and to verify the physicochemical and sensorial characteristics of the fruit liqueurs. The levels of moisture, dietary fiber, protein, lipids, ashes and carbohydrates and the energetic value of fruit pulp were quantified. Liqueurs of pulp and 
whole fruit were prepared with sugar concentrations of 150 and $250 \mathrm{~g} . \mathrm{L}^{-1}$. The liqueurs were submitted to sensory analysis of acceptance and purchase intention and determined the alcohol content, total acidity, $\mathrm{pH}$, soluble solids, color parameters, total phenolic compounds and antioxidant capacity. In the centesimal composition of the curriola pulp, the high fiber content is $12.75 \mathrm{~g} .100 \mathrm{~g}^{-1}$ and low calorie $54.11 \mathrm{kcal} .100 \mathrm{~g}^{-1}$. Significant differences $\mathrm{p}<0.05 \mathrm{were}$ observed in the liqueurs in the physical-chemical and sensorial analyzes of the liqueurs. Pulp liqueurs obtained a higher intensity of yellow with averages of $b^{*} 44.36$ and 44.42 and tended to color green with negative values of $a^{*}$ 0.89 and -1.87 , in contrast, whole fruit liqueurs presented a higher degree of red with positive values of a* 9.60 and 5.43. The color of pulp liqueurs was less accepted (4.23 and 4.32) in relation to the liqueurs of the whole fruit (5.46 and 5.46). The alcoholic concentrations of 20.58, 22.75, 33.58 and 42.75 influenced the overall impression, taste and intention to buy liqueurs. The liqueurs of the curriola fruit can reach better acceptance and purchase intention with the decrease of the alcoholic content.

Keywords: Pouteria ramiflora; Proximal composition; Liqueur; Acceptability; Alcoholic beverage.

\section{Resumen}

Este estudio tuvo como objetivo caracterizar la composición próxima del fruto de curriola (Pouteria ramiflora) y verificar las características fisicoquímicas y sensoriales de los licores de frutas. Se cuantificó el contenido de humedad, fibra dietética, proteínas, lípidos, cenizas e hidratos de carbono y el valor energético de la pulpa de la fruta. Se prepararon pulpa y licores de frutas enteras, con concentraciones de azúcares de 150 y $250 \mathrm{~g} . \mathrm{L}^{-1}$. Los licores fueron sometidos a análisis sensorial de aceptación e intención de compra y se determinó el contenido de alcohol, acidez total, $\mathrm{pH}$, sólidos solubles, parámetros de color, compuestos fenólicos totales y capacidad antioxidante. En la composición próxima de la pulpa de curriola destacan el alto contenido de fibra de $12,75 \mathrm{~g} .100 \mathrm{~g}^{-1}$ y el bajo en calorías $54,11 \mathrm{kcal} 100 \mathrm{~g}^{-1}$. Se observaron diferencias significativas $\mathrm{p}<0,05$ en los licores en los análisis físico-químicos y sensoriales. Los licores de pulpa tuvieron mayor intensidad de amarillo con medias de b* 44,36 y 44,42 y tendieron al color verde con valores negativos de $a^{*}-0,89$ y $-1,87$, por otro lado, enteros los licores de frutas mostraron un mayor grado de rojo con valores positivos de a* 9,60 y 5,43. El color de los licores de pulpa fue menos aceptado $(4,23$ y $4,32)$ en comparación con los licores de frutas enteras $(5,46$ y 5,46). Las concentraciones de alcohol de 20,58, 22,75, 33,58 y 42,75 influyeron en la impresión general, el gusto y la intención de compra de los licores. Los licores de frutas de curriola con menor grado alcohólico fueron los de mejor aceptación.

Palabras clave: Pouteria ramiflora; Composición próxima; Licor; Aceptabilidad; Bebida alcohólica.

\section{Introdução}

A currioleira (Pouteria ramiflora), pertencente à família Sapotaceae, tem sua exploração baseada, quase que exclusivamente, em extrativismo nas áreas de ocorrência natural, com seu fruto conhecida como curriola, brasa-viva, fruta-deveado, figo do cerrado, maçaranduba, pessegueiro-do-cerrado, pitomba-de-leite, e abiu-cutite (Coelho et al., 2009, SiBBr, 2019).

Os frutos curriola medem de 5 a $12 \mathrm{~mm}$ de diâmetro, possuem casca de coloração verde, mesmo quando maduros, cobrindo uma polpa branca, são de forma piriforme com uma semente coberta por uma polpa doce (Valente et al., 2013). A casca e a semente correspondem cerca de $57 \%$ do peso total do fruto. A porção comestível é constituída por $43 \%$ do fruto (Morzelle et al., 2015). Frutas são fundamentais para o homem e o meio ambiente, agregam valor à biodiversidade da Terra e a saúde humana, por serem alimentos funcionais e ricos em compostos bioativos e com um enorme potencial para sustentar e melhorar a segurança alimentar global, contribuindo para o bem-estar da humanidade (Petrović et al. 2021; Gecer et al., 2020). As bebidas alcoólicas destiladas apresentam um perfil do aroma suave, sendo uma das principais características devido aos seus múltiplos fatores, a presença de vários compostos voláteis identificados na análise destas bebidas. Há evidências de uma correlação positiva entre a capacidade antioxidante e o conteúdo de compostos fenólicos, sendo esses que auxiliam na prevenção de doenças devido a redução do estresse oxidativo e atividade de eliminação de radicais livres (Zhang et al., 2020; Franitza et al., 2016; Bhuyan, et al., 2015; Montero et al., 2020).

Licor é a bebida alcoólica produzida pela combinação de uma base alcoólica, aromas de frutas ou ervas com adição de xarope de açúcar, ácido cítrico e outros aditivos alimentares, bem como álcool e água, também podem ser de misturas de vários tipos de álcool, sucos, infusões de frutas e álcoois aromáticos obtidos pelo processamento de frutas, vale ressaltar que a preferência por determinado teor alcoólico dos licores está relacionada à combinação de sabor doce, teor alcoólico e sabor de 
fruta (Śliwińska et al., 2015; Abramova et al., 2020; Villa et al., 2021). Com características organolépticas típicas e uma grande variedade de sabores, desde o amargo até ao extremamente doce, com um aroma especial e cor diferente, que os tornam distintos em cada região (Montero et al., 2020). É um produto tradicional capaz de agregar valor à produção agrícola, sua fabricação é uma alternativa propícia para pequenos agricultores ou para grandes indústrias. A produção artesanal de licores é uma opção interessante para proporcionar aumento da renda familiar, pois além de agregar valor aos frutos desenvolvendo novo produto, o processamento requer tecnologia simples, o produto final é comercializado em temperatura ambiente e com maior tempo de prateleira (Villa et al., 2021). Devido aos teores de álcool e de açúcar, é possível elaborar um licor sem o emprego de conservantes, garantindo a preparação de um produto que seja ao mesmo tempo natural, estável e seguro (Teixeira et al., 2007). De acordo com o Ministério da Agricultura, Pecuária e Abastecimento [MAPA] (2009) licores são classificados como bebidas alcoólicas destiladas adocicadas e aromatizados sabores compatíveis, com teor alcoólico entre 15 e $54 \%$ de volume, a $20^{\circ} \mathrm{C}$ e teor de açúcar superior a $30 \mathrm{~g} . \mathrm{L}^{-1}$. A produção de licor vai além do preparo de uma bebida alcoólica simples há também o valor sustentável agregado tanto a saúde como a valorização dos frutos nativos (Silva et al., 2021). O uso sustentável da biodiversidade em programas relacionados à segurança alimentar e nutricional da população estimula a preservação e a conservação do bioma natural, como é recomendado pela Convenção sobre Diversidade Biológica (EsquinasAlcázar, 2005).

É importante dar prioridade às pesquisas com espécies nativas, em virtude da grande coleção de plantas não cultivadas, com boa aceitação comercial e que ainda são exploradas de forma extrativista, sistema que pode resultar na sua extinção, frente à ação do ser humano com desmatamento e contaminação dos solos (Silva et al., 2008).

Deste modo, o objetivo deste estudo foi de caracterizar a composição centesimal do fruto da currioleira (Pouteria ramiflora) do cerrado goiano, e verificar a aceitabilidade sensorial e as características físico-química de licores preparados com diferentes componentes do fruto, utilizados como forma de aproveitamento tecnológico.

\section{Metodologia}

As amostras de curriola (Pouteria ramiflora) compreenderam em polpa artesanal e frutos inteiros que foram adquiridos em janeiro de 2017 de fornecedor local. Os frutos eram provenientes da cidade de Aragarças, Goiás, Brasil, localizada a $15^{\circ} 53^{\prime} 51 " \mathrm{~S}$ de latitude e a $52^{\circ} 15^{\prime} 03^{\prime \prime} \mathrm{W}$ e longitude e altitude de 310 metros. As amostras de frutos e polpa congeladas foram transportadas em embalagem térmica refrigerada para Cuiabá, MT, Brasil.

\subsection{Análise de composição centesimal}

As análises da composição centesimal da parte comestível do fruto referente à umidade (012/IV), cinzas (018/IV) e lipídios (032/IV) foram determinadas conforme metodologia descrita pelo Instituto Adolfo Lutz [IAL] (2008). A quantificação de fibras dietética totais foi determinada segundo a Association of Oficial Analytical Collaboration International [AOAC] (1997), seção 45.4.07, método 985.29 e o teor de proteína total descrita na seção 12.1.07, método 960.52 (AOAC, 1997), multiplicando o conteúdo de nitrogênio total pelo fator de conversão 5,75, de acordo com a RDC $\mathrm{n}^{\circ} 360$, do Ministério da Saúde [MS] (2003). O teor de carboidratos totais foi estimado por diferença entre $100 \%$ e o somatório dos constituintes da composição centesimal (umidade, cinzas, lipídios, proteínas e fibra), conforme a RDC n 360 (Brasil, 2003). O valor calórico total foi calculado empregando-se fatores de Atwater, $4 \mathrm{kcal.g}{ }^{-1}$, para proteínas e carboidratos, e $9 \mathrm{kcal}^{-\mathrm{g}^{-1}}$, para lipídios (Brasil, 2003). 


\subsection{Obtenção dos licores}

Os frutos foram selecionados e sanitizados separados para análises físico-químicas e para o processamento dos licores de acordo com o fluxograma da Figura 1.

Figura 1. Fluxograma de produção artesanal de licores de curriola (Pouteria ramiflora).
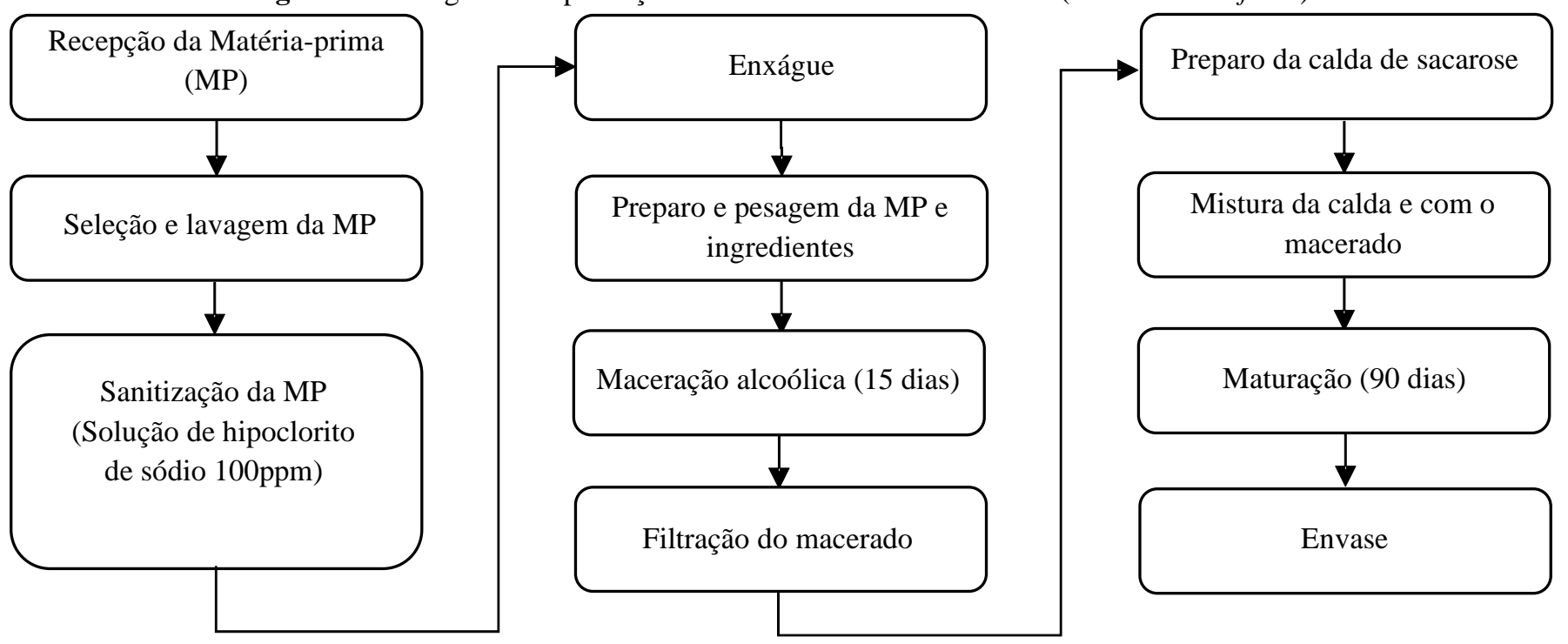

Fonte: Autores.

A elaboração dos licores foi realizada conforme o fluxograma da Figura 1 baseado nos procedimentos relatados por Venturini (2010), no qual para a maceração foi utilizada na proporção de polpa ou fruto de 1:2 com a cachaça comercial. Cachaça e açúcar refinado utilizados foram adquiridos no comércio local. Pesou-se oito porções de polpa de quinhentos gramas, as polpas foram colocadas sob infusão em porções de um litro de cachaça em potes de vidro fechado hermeticamente. Os frutos inteiros (casca, polpa e semente) foram cortados ao meio e pesados em oito porções de quinhentos gramas e colocados em um litro de cachaça foram colocados em potes de vidro fechado hermeticamente. As formulações foram colocadas em maceração por 15 dias em local protegido da luz. Após a maceração realizou-se a filtração em peneira de material inoxidável com orifícios de $3 \mathrm{~mm}$ e com o auxílio de tecido tipo feltro, diminuindo a presença de resíduos.

Após esta etapa procedeu-se o cálculo dos volumes de água e açúcar para a redução do teor alcoólico do macerado, conforme as equações 1 e 2 e procedeu-se ao preparo calda de sacarose com água potável, em quantidades que no produto final houvesse uma concentração de 150 e $250 \mathrm{~g}$ de açúcar para cada litro de licor. A calda, à temperatura ambiente, foi adicionada ao filtrado, evitando a perda de álcool por evaporação.

$$
\begin{gathered}
V_{H_{2} O}=\frac{V_{m} \times T A_{i}}{T A_{f}}-V_{m} \\
m=V_{f} \times[a]
\end{gathered}
$$

Onde: $\quad \mathrm{V}_{\mathrm{H} 2 \mathrm{O}}$ - Volume de água

$\mathrm{V}_{\mathrm{m}}$ - Volume do macerado

$\mathrm{TA}_{\mathrm{i}}$ - Teor Alcoólico inicial.

$\mathrm{TA}_{\mathrm{f}}$ - Teor Alcoólico final. 


\author{
$\mathrm{V}_{\mathrm{f}}$ - Volume final do Licor. \\ $\mathrm{m}$ - Massa da sacarose \\ [ $a$ ] - Concentração de sacarose.
}

Após a adição da calda, o licor foi homogeneizado e deixado em repouso por 90 dias em local escuro à temperatura ambiente. Posteriormente, os licores foram envazados em garrafas de vidro com capacidade de $900 \mathrm{~mL}$ e vedados com rolha de cortiça.

\title{
2.3 Análises químicas e físico-químicas dos licores
}

Os índices de teor alcoólico (217/IV), pH (017/IV), acidez total (235/IV) e sólidos solúveis (315/IV) foram avaliados conforme os métodos de análises de alimentos (IAL, 2008). Os parâmetros de cor foram avaliados pelo sistema CIE L* $\mathrm{a}^{*} \mathrm{~b}^{*}$, luminosidade ( $\left.\mathrm{L}^{*}\right)$, índice de vermelho ( $\mathrm{a}^{*}$, índice de vermelho) e índice de amarelo ( $\mathrm{b}^{*}$, índice de amarelo), obtidos utilizando um colorímetro CM700D (Konica Minolta, Incorporation) calibrado para um padrão branco, iluminante D65, 10 graus para observador padrão e componente especular excluído (SCE). Foram realizadas três leituras em cubeta de vidro 10mm (CMA98).

O conteúdo dos compostos fenólicos nos licores foi determinado pelo método de Folin-Ciocalteu, usando o ácido gálico como padrão. A reação ocorreu com $0,5 \mathrm{~mL}$ de cada licor, $2,5 \mathrm{~mL}$ de reagente de Folin-Ciocalteu diluído a 1:10 em água destilada e $2 \mathrm{~mL}$ de solução a $4 \%$ (m:v) de carbonato de sódio. A absorbância foi medida a 740nm. Os resultados dos fenólicos totais foram expressos como $\mu \mathrm{g}$ de equivalente de ácido gálico (GAE). $\mathrm{mL}^{-1}$ de licor (Woisky \& Salatino, 1998).

As amostras foram avaliadas em termos de liberação de hidrogênio ou capacidade de eliminação de radicais usando o radical estável 2,2-difenil-1-picril hidrazil (DPPH) (Brand-Williams et al., 1995). Resumidamente, na reação de mistura continha $500 \mu \mathrm{L}$ da amostra e $300 \mu \mathrm{L}$ da solução de DPPH. A absorbância da mistura reacional foi medida a $517 \mathrm{~nm}$ contra o branco, que não continha amostra. Os resultados foram expressos em $\mu \mathrm{g}$ Trolox. $\mathrm{mL}^{-1}$ de licor, e os valores de equivalência total foram calculados usando a curva padrão de Trolox de 0 a $25 \mu \mathrm{g} \cdot \mathrm{mL}^{-1}$.

\subsection{Análise sensoriais dos licores}

O projeto para análise sensorial foi aprovado pelo Comitê de Ética em Pesquisa IFMT no processo 75509417.0.0000.8055. A aceitação e a intenção de compra foram realizadas por 119 avaliadores maiores de dezoito anos, compostos pela comunidade acadêmica da Universidade Federal de Mato Grosso, entre eles alunos e servidores. Com o objetivo de conhecer o grupo de avaliadores, estes foram orientados a preencher uma ficha do perfil do avaliador, com questionário quanto ao gênero, faixa etária e hábitos de consumo de licor e os resultados apresentados em percentuais.

As amostras de licor, cerca de $20 \mathrm{~mL}$, foram codificados com três dígitos e avaliadas de forma monádica, em blocos completos balanceados, em cabines individuais, sob luz branca. Para a análise de aceitação da cor, aroma, textura, sabor e impressão global foi utilizada escala não estruturada de nove centímetros, nos seus extremos "desgostei extremamente" $\mathrm{e}$ "gostei extremamente". Para a intenção de compra foi utilizada uma escala verbal com cinco termos, de "certamente compraria" a "certamente não compraria" (Minim, 2013). A frequência das respostas foi distribuída em gráfico de barras.

\subsection{Delineamento experimental e análise estatística}

O experimento foi conduzido em Delineamento em Blocos Casualizados (DBC), disposto por um fatorial 2 × 2, duas fontes de aroma (polpa e fruto integral) e duas concentrações de açúcar (150 e $250 \mathrm{~g} . \mathrm{L}^{-1}$ ), sendo quatro repetições cada uma. 
Adotou-se como uma unidade experimental uma garrafa de $900 \mathrm{~mL}$ das diferentes formulações do licor de curriola.

As médias obtidas na análise físico-química e de aceitação sensorial dos licores foram submetidas ao tratamento estatístico de análise da variância e teste de Tukey com nível de significância de (p>0,05) pelo programa estatístico PAST 2.17c (Hammer et al., 2001).

\section{Resultados e Discussão}

A composição centesimal de polpas de frutos in natura possibilita o conhecimento do valor calórico e das porções de cada componente em $100 \mathrm{~g}$ da porção comestível do alimento. Os resultados da composição centesimal da polpa de curriola podem ser observados na Tabela 1 .

Tabela 1. Composição centesimal $\left(\mathrm{g} \cdot 100 \mathrm{~g}^{-1}\right)$, valor energético total e dados da literatura relativos à polpa in natura de curriola (Pouteria ramiflora).

\begin{tabular}{lcc}
\hline Componente $\left(\mathrm{g} .100 \mathrm{~g}^{-1}\right)^{1}$ & Polpa & Morzelle et al. 2015 \\
\hline Umidade & $73,69 \pm 0,24$ & $70,68 \pm 1,52$ \\
Fibra alimentar total & $12,75 \pm 0,03$ & $8,18 \pm 1,08^{3}$ \\
Proteína (N x 5,75) & $1,50 \pm 0,05$ & $2,08 \pm 0,01$ \\
Lipídios & $0,44 \pm 0,08$ & $0,84 \pm 0,01$ \\
Cinzas & $0,58 \pm 0,02$ & $1,06 \pm 0,03$ \\
Carboidratos & \\
\hline Valor energético total & 11,04 & 17,16 \\
(kcal.100g $\left.{ }^{-1}\right)$ & 54,11 & - \\
\hline $\begin{array}{l}{ }^{1} \text { Os valores constituem médias } \pm \text { desvios-padrão de três repetições, exceto } \\
\text { fibra alimentar total, analisada em quatro repetições. }{ }^{2} \mathrm{O} \text { valor energético } \\
\text { total não está disponível na literatura citada. }{ }^{3} \text { Fibra bruta. }\end{array}{ }^{4}$ Os carboidratos \\
totais foram calculados por diferença. Fonte: Autores.
\end{tabular}

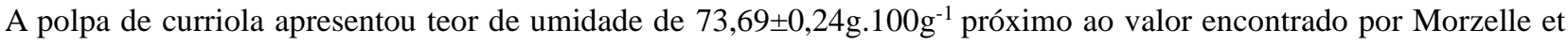
al. (2015) de 71\% em polpa de curriola da mesma região. Em estudo da composição centesimal de onze frutos nativos do cerrado (macaúba, caju-do-cerrado, araticum, murici, gabiroba, cagaita, mangaba, puçá, araçá, chichá e pitomba) Silva et al. (2008) obtiveram média de 73\% de umidade e Espírito Santo et al. (2020) encontrou resultados diferentes com 79,62 $\pm 0,35$ na polpa de Pouteria glomerata frescos e superior aos encontrados nos frutos do gênero Pouteria sapota Jacq tendo obtido 63,8 g.100g $\mathrm{g}^{-1}$ (Yahia \& Gutiérrez-Orozco, 2011). A quantificação de umidade é importante, pois, pode influenciar na suculência, na composição, na qualidade e na estabilidade dos frutos (Silva et al., 2018). A polpa apresentou teor elevado de fibra alimentar $12,75 \pm 0,03 \mathrm{~g} .100 \mathrm{~g}^{-1}$, o valor encontrado neste estudo difere se ao encontrado em estudo de estruturas químicas da fibra alimentar e propriedades físico-químicas de frutas comestíveis da Pouteria glomerata nativas do pantanal brasileiro cujo o resultado de teor de fibras alimentares, foi maior o rendimento de fibras alimentares insolúveis com (52\%) do que solúveis (8\%) obtido é fruta de P. glomerata liofilizada (Espírito Santo et al., 2020). Segundo a legislação brasileira do Ministério da Saúde (MS, 1998), um alimento deve conter no mínimo de 6\% (m:m) de fibra alimentar para ser considerado com alto teor de fibras e o International Life Sciences Institute do Brasil [ILSI Br] (2003) recomenda uma ingestão média diária de 25g de fibras para mulheres e $38 \mathrm{~g}$ para homens entre as faixas etárias de 19 a 50 anos. O teor de fibras encontrado na polpa de curriola é cerca de duas vezes maior se comparados com alguns frutos populares no Brasil como, abacate, pera Park e goiaba com 
casca, os quais possuem em média 6,3\% (m:m) de fibra alimentar segundo o Núcleo de Estudos e Pesquisas em Alimentação [NEPA] (2011), que também são considerados com alto teor de fibras.

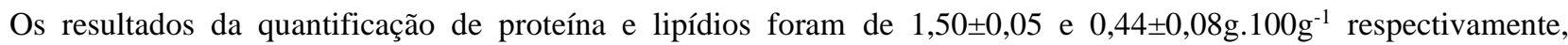
quantidades aproximadas às avaliadas por Morzelle (2015) de 2 e 0,8\% no mesmo fruto. O teor de proteína achado nesse estudo é superior ao encontrado por Espírito Santo et al., (2020) na polpa dos frutos da espécie Pouteria glomerato fresca que 1,41g. $100 \mathrm{~g}^{-1}$ e o resultado de lipídios da polpa do fruto de curriola analisado neste estudo foi inferior em relação aos frutos frescos de P. glomerata que teve como resultado 0,79g.100 $\mathrm{g}^{-1}$ (Espírito Santo et al., 2020).

O teor de cinzas quantificado na polpa de curriola foi de $0,58 \pm 0,02 \mathrm{~g} .100 \mathrm{~g}^{-1}$, em estudo do fruto Morzelle et al. (2015) encontrou $1 \%$ para o mesmo componente diferente do encontrado em gênero de $P$. glomerata com $0,70 \pm 0,01 \mathrm{~g} .100 \mathrm{~g}^{-1}$ (Espírito Santo et al., 2020). O conteúdo de cinzas está relacionado com a quantidade de matéria mineral na amostra, solos distintos, suas composições, fertilidade do solo, fatores genéticos e condições ambientais em que a planta se cresceu podem interferir nas diferenças de teor de cinzas de frutos (Silva et al., 2016).

A polpa de curriola apresentou $11,04 \mathrm{~g} \cdot 100 \mathrm{~g}^{-1}$ de carboidratos, semelhante ao estudo de Schiassi et al. (2018) que avaliou seis frutos do cerrado (araçá, buriti, cajá-amarelo, cagaita, mangaba e marolo) que tiveram em média de $10 \%$ de carboidratos e menor quantidade de carboidratos achado nesse estudo comparando com o conteúdo total de carboidratos de $\left(17,42 \mathrm{~g} .100 \mathrm{~g}^{-1}\right)$ encontrados nos frutos frescos de P. glomerata (Espírito Santo et al., 2020).

Assim, com os valores de carboidratos, proteínas e lipídeos foi calculado valor energético resultando em 54,11 $\mathrm{kcal} 100 \mathrm{~g}^{-1}$, resultado semelhante ao encontrado por Silva et al. (2008) em murici, gariroba e pitomba $(46,43,47,36$ e 56,35 kcal. $\left.100 \mathrm{~g}^{-1}\right)$.

Os licores de curriola foram elaborados como descrito anteriormente, em seguida, submetidos a análises físicoquímicas e avaliação sensorial. Os resultados das análises físico-químicas dos licores estão apresentados na Tabela 2.

Tabela 2. Características físico-químicas de licores de curriola (Pouteria ramiflora) de polpa e fruto integral e concentrações de açúcar de 150 e $250 \mathrm{~g} . \mathrm{L}^{-1}$.

\begin{tabular}{|c|c|c|c|c|}
\hline \multirow[b]{2}{*}{ Características $^{1}$} & \multicolumn{2}{|c|}{ Licor de polpa } & \multicolumn{2}{|c|}{ Licor de fruto } \\
\hline & 150g.L $\mathrm{L}^{-1}$ & $250 \mathrm{~g} \cdot \mathrm{L}^{-1}$ & 150g.L $\mathrm{L}^{-1}$ & $250 \mathrm{~g} / \mathrm{L}^{-1}$ \\
\hline $\begin{array}{r}\text { Teor alcoólico }(\% \mathrm{v} / \mathrm{v} \\
\left.20^{\circ} \mathrm{C}\right)\end{array}$ & $20,58 \pm 0,63^{\mathrm{aA}}$ & $22,75 \pm 0,87^{\mathrm{bB}}$ & $33,58 \pm 0,31^{\mathrm{cC}}$ & $42,75 \pm 0,56^{\mathrm{dD}}$ \\
\hline PH & $4,77 \pm 0,02^{a}$ & $4,79 \pm 0,02^{\mathrm{a}}$ & $5,27 \pm 0,11^{\mathrm{bA}}$ & $5,63 \pm 0,10^{\mathrm{cB}}$ \\
\hline $\begin{array}{r}\text { Acidez total }(\mathrm{g} \mathrm{NaOH} \\
\left.100 \mathrm{~mL}^{-1}\right)\end{array}$ & $0,80 \pm 0,01^{\mathrm{aA}}$ & $0,34 \pm 0,01^{\mathrm{bB}}$ & $0,18 \pm 0,01^{\mathrm{cC}}$ & $0,11 \pm 0,01 \mathrm{dD}$ \\
\hline $\begin{array}{r}\text { Sólidos solúveis } \\
\left({ }^{\circ} \text { Brix }\right)\end{array}$ & $21,24 \pm 0,71^{\mathrm{aA}}$ & $29,24 \pm 0,47^{\mathrm{bB}}$ & $27,23 \pm 0,63^{\mathrm{bC}}$ & $34,14 \pm 2,22 \mathrm{cD}$ \\
\hline $\mathbf{L}^{*}$ & $49,39 \pm 0,14^{\mathrm{aA}}$ & $50,90 \pm 0,31^{\mathrm{aB}}$ & $45,06 \pm 0,81^{\mathrm{bC}}$ & $49,60 \pm 1,25^{\mathrm{aD}}$ \\
\hline $\mathbf{a}^{*}$ & $-0,89 \pm 0,03^{\mathrm{aA}}$ & $-1,87 \pm 0,12^{\mathrm{bB}}$ & $9,65 \pm 0,32^{\mathrm{cC}}$ & $5,43 \pm 0,49 \mathrm{dD}$ \\
\hline $\mathbf{b}^{*}$ & $44,36 \pm 0,47^{\mathrm{a}}$ & $44,42 \pm 0,32^{\mathrm{a}}$ & $32,78 \pm 0,26^{b}$ & $26,93 \pm 0,46^{c}$ \\
\hline $\begin{array}{r}\text { Compostos fenólicos } \\
\text { totais }\left(\mathrm{mg} \mathrm{GAE} \mathrm{L}^{-1}\right)^{2} \\
\text { Capacidade }\end{array}$ & $376,15 \pm 6,39^{a}$ & $361,88 \pm 17,73^{\mathrm{a}}$ & $672,28 \pm 21,03^{\mathrm{bA}}$ & $397,05 \pm 21,60^{\mathrm{aB}}$ \\
\hline $\begin{array}{r}\text { antioxidante (mg } \\
\left.\text { Trolox } \mathbf{L}^{-1}\right)\end{array}$ & $114,43 \pm 8,20^{a}$ & $88,47 \pm 35,19^{a}$ & $337,45 \pm 34,13^{b A}$ & $211,30 \pm 8,63^{\mathrm{cB}}$ \\
\hline
\end{tabular}

${ }^{1}$ Médias e desvio padrão seguidas pela mesma letra minúsculas nas linhas não diferem estatisticamente pelo teste de Tukey (p>0,05). Diferentes letras maiúsculas nas linhas denotam diferença estatística entre os licores de mesma fonte de aroma pelo teste de Tukey (p>0,05). ${ }^{2}$ Equivalente de ácido gálico (GAE). $\mathrm{L}^{-1}$. Fonte: Autores. 
O teor alcoólico dos licores de curriola mantiveram-se dentro dos limites preconizados pela legislação para o produto, que é entre 15 e $54 \%$ volume por volume a $20^{\circ} \mathrm{C}$ (MAPA, 2009), variando com diferença significativa de 20,58 $\pm 0,63$ a $42,75 \pm 0,56 \%$ volume por volume a $20^{\circ} \mathrm{C}(\mathrm{p}>0,05)$, na bebida destilada de morangos envelhecida em madeira teve resultados de teor alcoólico nas suas formulações variando de 46,8 e 47,9\%, sendo valores superiores aos encontrados nesse estudo (Anjos et al., 2021). E valores próximos foram achados por Leonarski et al., (2021) em seu trabalho de desenvolvimento e caracterização de licor de três diferentes frutas nativas brasileiras (jabuticaba, amora-preta e guabiroba) tendo variado de

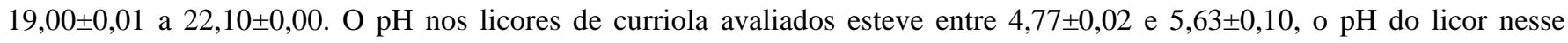
estudo foi superior ao encontrado por Anjos et al. (2021) na caracterização de uma bebida destilada produzida com frutos de morangueiro (Arbutus unedo L.) e envelhecida em madeira de carvalho em escala laboratorial, em diferentes formulações cujo

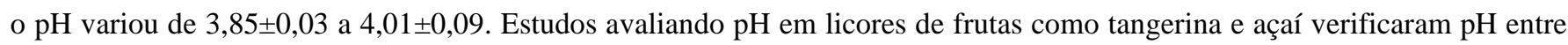
4,77 e 3,52 respectivamente (Almeida et al., 2012; Oliveira \& Santos, 2011), a variação é decorrente da diferença da composição da matéria-prima utilizada no processamento dos licores.

Outrossim, segundo Teixeira et al. (2012), licores com pH inferior a 4,5 junto com teor alcoólico acima de 12\% formam um fator positivo para preservação da bebida.

A acidez total em gramas de $\mathrm{NaOH} .100 \mathrm{~mL}^{-1}$ variou entre de $0,11 \pm 0,01$ no licor de fruto integral e $250 \mathrm{~g} . \mathrm{L}^{-1}$ de açúcar e $0,38 \pm 0,01$ no licor de polpa e $150 \mathrm{~g} . \mathrm{L}^{-1}$ de açúcar, os valores de acidez total deste estudo se apresentaram acima dos valores de acidez fixa das formulações de bebida destilada produzida com frutos de morangueiro (Arbutus unedo L.) encontrados por Anjos et al. (2021) cujo os resultados variaram de 0,009 $\pm 0,001 *$ (bebida controle) a 0,083 $\pm 0,005$, mas inferiores a valores de acidez fixa da formulação MT6 (MT6 - envelhecido em madeira torrada e média durante seis meses) com 0,105 $\pm 0,006$. Os valores de acidez total deste estudo foram inferiores em relação aos valores de acidez total analisados por Anjos et al. (2021)

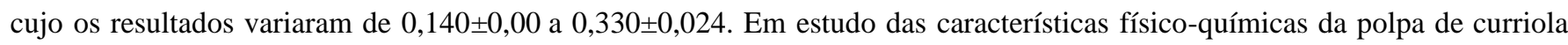
de Morzelle et al. (2015) a acidez titulável foi 0,12\%, resultado aproximado para os licores de fruto integral.

Possivelmente a variação de acidez e pH estejam relacionadas com a dissolução dos ácidos orgânicos durante os quinze dias de maceração alcoólica, pois a área de contato da polpa com o álcool é maior em relação ao fruto integral, facilitando a dissolução dos ácidos, fazendo com que aumentasse a acidez e abaixasse o pH, o pH ácido é um fator limitante importante para o crescimento de microrganismos patogênicos e em deterioração nos alimentos e contribui para a longa tempo de prateleira do alimento (Leonarski et al., 2021; Silva et al., 2016).

O percentual de sólidos solúveis nos licores de curriola variou de $21,24 \pm 0,71$ a $34,14 \pm 2,22^{\circ}$ Brix, com diferenças significantes ( $p>0,05)$, entre amostras e entre os grupos licor de polpa e de fruto integral, resultados próximos foram achados por Leonarski et al., (2021) em seu trabalho de desenvolvimento e caracterização de licor de três diferentes frutas nativas

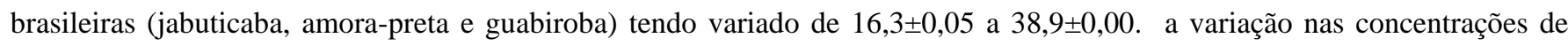
sólidos solúveis nas formulações de licor pode ser explicado como a consequência de concentração inicial de açúcares e outros sólidos solúveis naturalmente ocorrendo na fruta, e até mesmo as características das matérias-primas, pois os sólidos solúveis são o total de todos os sólidos dissolvidos incluindo açúcar, sais, proteínas e ácidos entre outros (Leonarski et al., 2021; Silva et al., 2016).

A coloração do licor foi avaliada nos parâmetros de $\mathrm{L}^{*}, \mathrm{a}^{*}$ e b*, separadamente. Quanto à luminosidade (L*), a

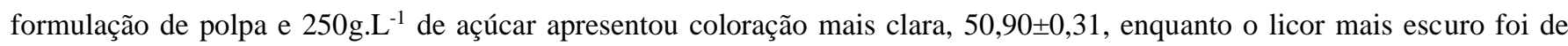
fruto e $150 \mathrm{~g} . \mathrm{L}^{-1}$ com valor de $\mathrm{L} * 45,06 \pm 0,81$. Para a coordenada $\mathrm{a}^{*}$ os licores do fruto integral apresentaram valores positivos

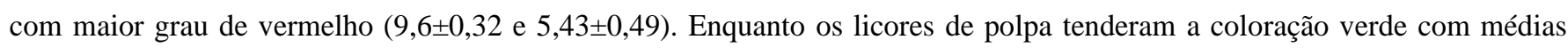
negativas para $a^{*}(-0,89 \pm 0,03$ e $-1,87 \pm 0,12)$. Para $b^{*}$, notou-se maior intensidade do amarelo para os licores de polpa com

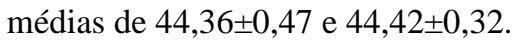


Observou-se uma coloração diferenciada dos licores de fruto integral e de polpa, essa diferença pode ser atribuída a matéria-prima, pois nos licores de polpa o álcool interagiu somente com a polpa do fruto, enquanto na maceração do licor de fruto inteiro o álcool extraiu parte da cor da casca e principalmente da semente, resultando numa coloração castanho-claro ao castanho-escuro, enquanto os licores de polpa apresentaram cor amarelada e esverdeada.

Os licores de fruto integral apresentaram teor de compostos fenólicos totais de $672,28 \pm 21,03$ e $397,05 \pm 21,60 \mathrm{mg}$

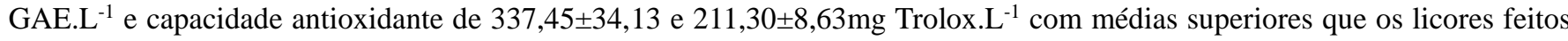

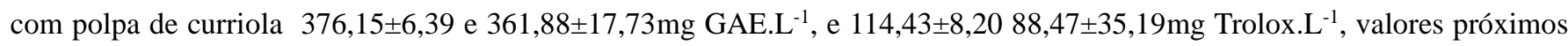

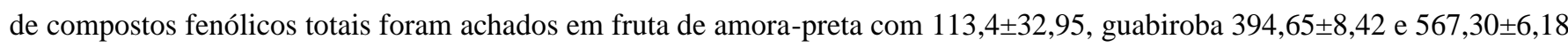
em jabuticaba (Leonarski et al., 2021). Esta diferença pode ser atribuída a interação da fonte alcoólica com a casca e a semente do fruto, como relatou Roesler et al. (2007) que os compostos fenólicos e a atividade antioxidante em extratos de semente e casca de araticum, de semente de cagaita, de cascas de pequi e de banha de galinha se sobrepuseram aos extratos de polpa desses frutos.

Desta forma diferentes fontes de aroma de licores resultam em quantidades diferentes de compostos bioativos. Serreli et al. (2017) em estudo de licores de mirto branco de Cagliari, Itália quantificou 636mg GAE.L ${ }^{-1}$ de compostos fenólicos e 930mg Trolox.L ${ }^{-1}$ de capacidade antioxidante. Na Silésia - Polônia, Polak \& Bartoszek (2015) avaliaram 10 licores de amora, framboesa, cereja, especiarias, nozes, sabugueiro e sorbus encontrando em média de $861 \mathrm{mg}$ GAE.L. ${ }^{-1}$ e 566mg Trolox.L $L^{-1}$, essa variação também depende dos compostos fenólicos específicos presentes nas diferentes frutas.

A análise sensorial dos licores de curriola contou com 119 avaliadores, apresentando uma maioria de jovens $84 \%$ (Figura 2A) entre 18 e 25 anos de idade e de mulheres 61\% (Figura 2B). Quando questionado aos avaliadores, se consomem licores, 55\% declararam que consomem (Figura 2C), destes consumidores 54\% disseram consumir uma vez por semestre e somente $23 \%$ consomem ao menos uma vez por mês (Figura 2D). 
Figura 2. Distribuição dos 119 avaliadores de licores de curriola (Pouteria ramiflora) quanto ao gênero (A); Distribuição quanto a idade dos avaliadores (B); Distribuição do consumo de licores (C); Distribuição quanto a frequência de consumo de licores (D).

A
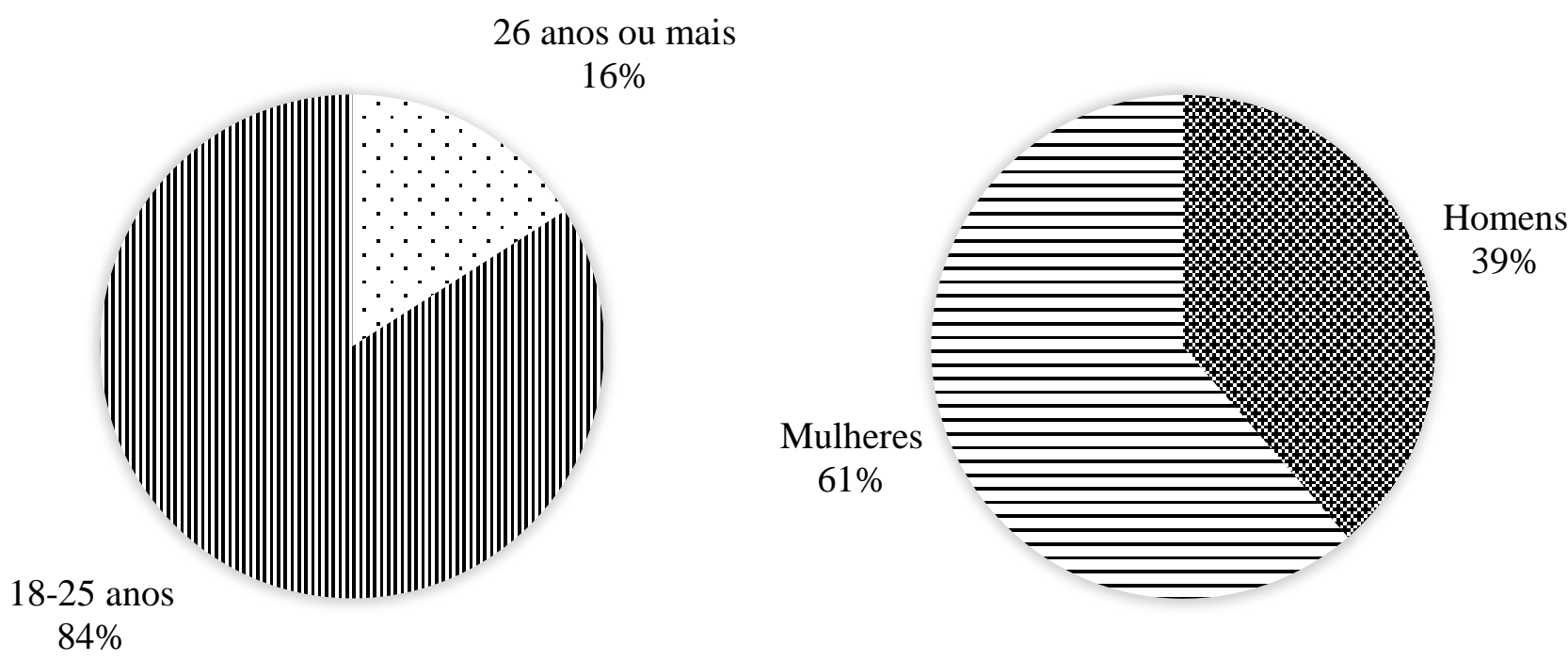

$84 \%$

$\mathrm{C}$
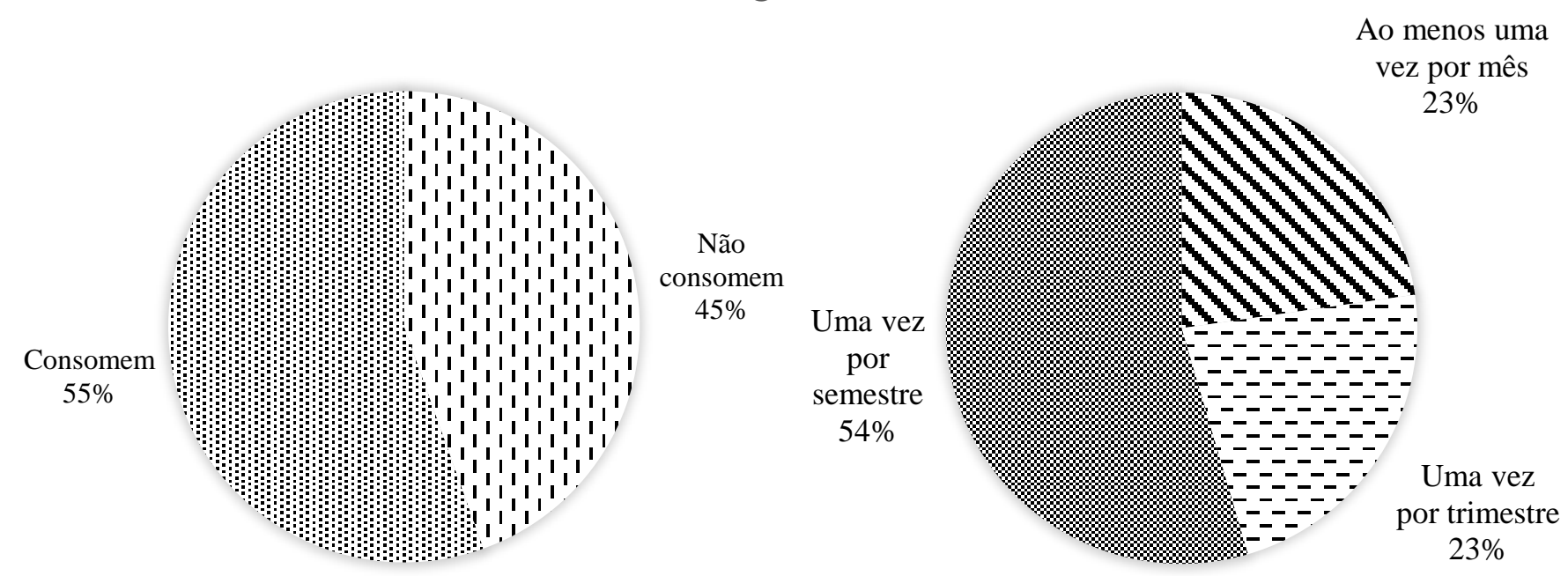

Fonte: Autores.

Os valores médios de aceitação dos licores estão apresentados na Tabela 3. 
Tabela 3. Valores médios de aceitação de licores de curriola (Pouteria ramiflora) de polpa e fruto integral e concentrações de açúcar de 150 e $250 \mathrm{~g} . \mathrm{L}^{-1}$.

\begin{tabular}{ccccccc}
\hline $\begin{array}{c}\text { Fonte de } \\
\text { aroma }\end{array}$ & $\begin{array}{c}\text { Concentração de } \\
\text { açúcar }\end{array}$ & Cor & Aroma & Textura & Sabor & Impressão global \\
\hline Polpa & $150\left(\mathrm{~g} \cdot \mathrm{L}^{-1}\right)$ & $4,32^{\mathrm{a}}$ & 4,96 & 5,78 & $5,53^{\mathrm{aA}}$ & $5,23^{\mathrm{a}}$ \\
Polpa & $250\left(\mathrm{~g} \cdot \mathrm{L}^{-1}\right)$ & $4,23^{\mathrm{a}}$ & 4,85 & 5,84 & $6,09^{\mathrm{aB}}$ & $5,57^{\mathrm{a}}$ \\
Fruto & $150\left(\mathrm{~g} \cdot \mathrm{L}^{-1}\right)$ & $5,68^{\mathrm{b}}$ & 5,44 & 5,45 & $4,56^{\mathrm{b}}$ & $5,04^{\mathrm{bA}}$ \\
Fruto & $250\left(\mathrm{~g} \cdot \mathrm{L}^{-1}\right)$ & $5,46^{\mathrm{b}}$ & 5,22 & 5,19 & $4,16^{\mathrm{b}}$ & $4,42^{\mathrm{bB}}$ \\
\hline
\end{tabular}

${ }^{1}$ Médias seguidas por diferentes letras minúsculas nas colunas diferem-se estatisticamente pelo teste de Tukey (p>0,05). Diferentes letras maiúsculas nas linhas denotam diferença estatística entre os licores de mesma fonte de aroma pelo teste de Tukey (p>0,05). Fonte: Autores.

A análise sensorial da aceitação dos aromas não apresentou diferença significativa $(p>0,05)$ entre os licores de polpa ou fruto integral nas concentrações de açúcar 150 e $250 \mathrm{~g} . \mathrm{L}^{-1}$. Assim, a fonte do aroma sendo a polpa ou o fruto integral não interferiu no grau de aceitação dos aromas dos licores.

Quanto às texturas dos licores, embora as concentrações de açúcar possam alterar a viscosidade de soluções, as concentrações de açúcar 150 e $250 \mathrm{~g} . \mathrm{L}^{-1}$ não alteraram a aceitação sensorial da textura sentida pelos avaliadores no grau de significância $(\mathrm{p}>0,05)$.

A cor mais aceita foi a dos licores de fruto integral com médias de 5,68 e 5,46 enquanto os de licores de polpa apresentaram 4,32 e 4,23. A melhor aceitação dos licores de fruto integral pode ser atribuída a sua cor castanha contra a coloração amarela e esverdeada dos licores de polpa. Segundo Teixeira (2009), a cor e a aparência é o primeiro contato do avaliador com um produto, as reações individuais de aceitação de um produto estão associadas a uma aparência e uma cor esperadas. Se as características forem muito diferentes da forma natural ou comercial podem causar rejeição.

Foram observadas diferenças significativas na aceitação dos atributos sabor e impressão global com maior aceitabilidade para os licores de polpa de curriola. A concentração de açúcar dos licores de polpa interferiu significativamente no atributo sabor, resultando em média de 6,09 para o licor de polpa mais doce. As médias de análise sensorial atribuídas pelos

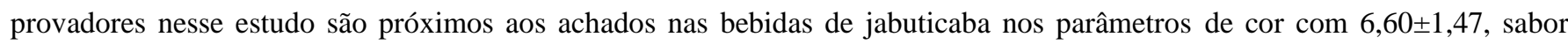

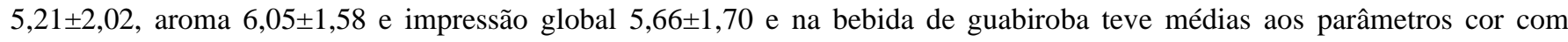

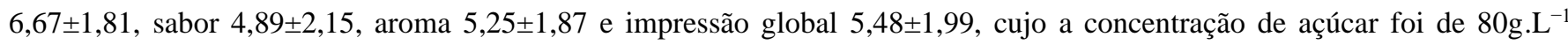
(Leonarski et al., 2021).

O licor de fruto integral com maior concentração de açúcar foi a amostra com menor aceitação na impressão global 4,42, possivelmente o teor alcoólico de cerca de $43 \%$ tenha contribuído para o aroma e sabor menos aceite e ainda tenha sido o responsável pela baixa aceitação. O aroma estável desempenha um papel crítico na aceitação do consumidor e contribui significativamente para a decisão das bebidas além de que, os compostos fenólicos presentes nas bebidas à base de frutas contribuem para o sabor agradável pela complexa natureza dos perfis voláteis, a composição volátil que muda continuamente nas frutas durante o processo de maturação.

Observa-se na Figura 3 a distribuição da frequência da intenção de compra dos licores de curriola. Os licores produzidos com polpa da fruta obtiveram maior nível de intenção de compra em relação aos licores de fruto integral. Foram atribuídas ao licor de polpa com mais açúcar a melhor atitude de compra de certamente e provavelmente compraria em relação aos outros licores. 
Figura 3. Distribuição percentual de intenção de compra dos licores de curriola (Pouteria ramiflora) produzidos de polpa e do fruto integral e em concentrações de açúcar de 150 e $250 \mathrm{~g} . \mathrm{L}^{-1}$.

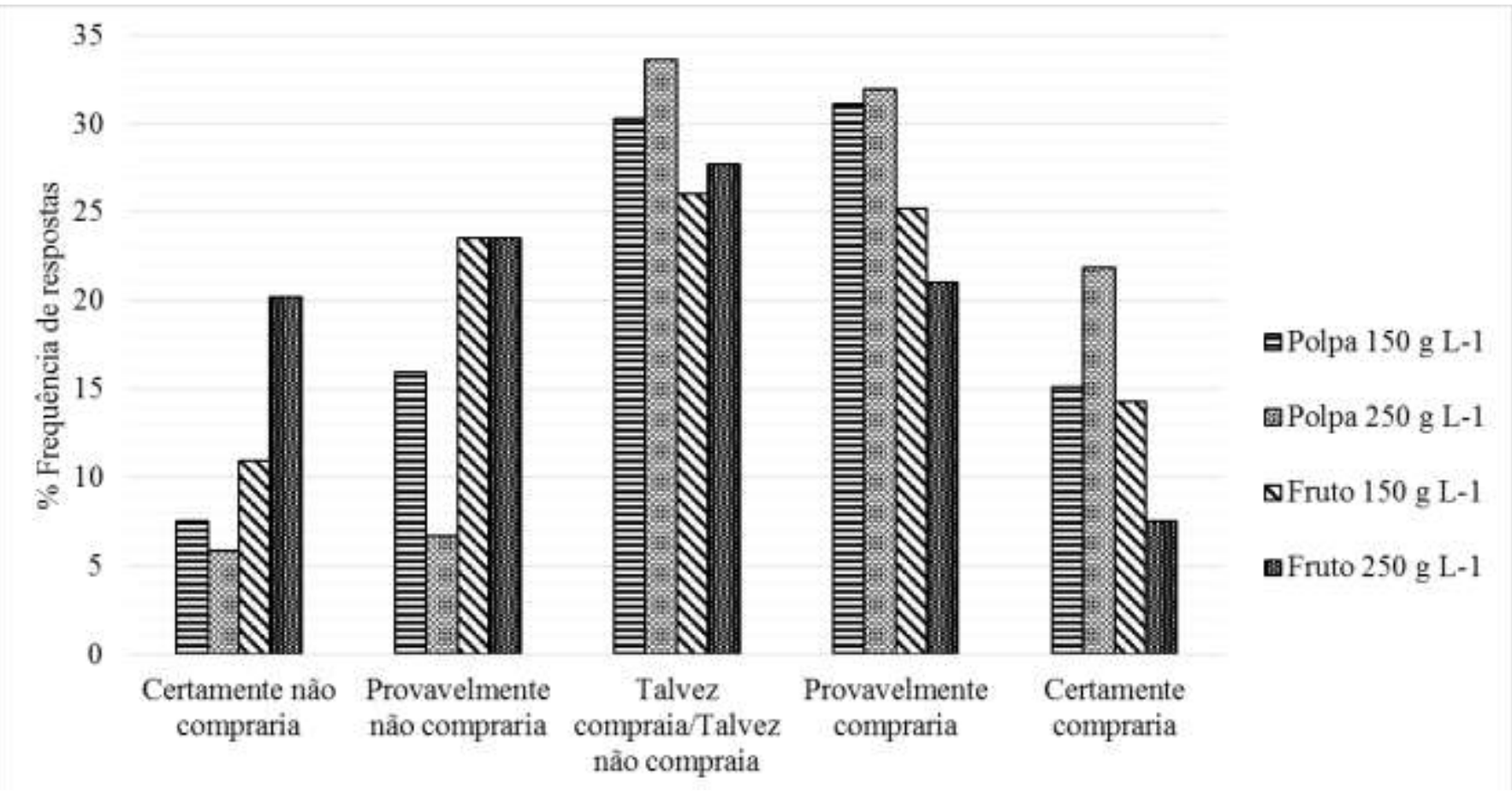

Fonte: Autores.

Possivelmente a concentração alcoólica dos licores influenciou na aceitação e atitude de compra dos mesmos. Estudos denotam que a diferença do teor alcoólico influencia diretamente na aceitação de bebidas. Embora a legislação brasileira para licores estabeleça limites entre 15 e $54 \%$ volume por volume a $20{ }^{\circ} \mathrm{C}$, autores tem trabalhado com teor alcoólico próximo a 20\%. Em trabalho sensorial de Odello \& Braceschi (2009) com vinte cachaças, os resultados dos métodos sensoriais descritivo e afetivo indicaram que a porcentagem alcoólica é uma das características decisivas para baixas classificações. Assim como em estudo de Oliveira et al. (2014) de licores de graviola com teor alcoólico de 15,0 a 16,7\% (V:V), a maior preferência foi para licores com menor graduação alcoólica.

\section{Conclusão}

A caracterização de uma bebida a partir deste fruto curriola (Pouteria Ramiflora) foi avaliado pela primeira vez. Os resultados revelam que este fruto é rico em fibras e com baixa concentração de carboidratos, proteínas, lipídios e calorias. As diferentes formulações de licores de curriola apresentaram diferenças significativas nos parâmetros físico-químicos e sensoriais avaliados. As diferentes formulações de licores de curriola apresentaram diferenças significativas nos parâmetros físicoquímicos e sensoriais avaliados. A padronização da concentração alcoólica dos licores de fruto de curriola é imprescindível para obter maior aceitação quanto ao sabor, impressão global e a intenção de compra. Em geral, a qualidade sensorial do licor teve aceitação por parte dos provadores.

\section{Agradecimentos}

Ao Instituto Federal de Educação, Ciência e Tecnologia de Mato Grosso (IFMT) pelo apoio financeiro junto projeto por meio da Instrução Normativa $n^{\circ} 10$ e Resolução $n^{\circ}$ 05/2015 de fomento a pós-graduação, principalmente à toda a equipe do Programa de Pós-graduação em Ciência e Tecnologia de Alimentos do IFMT, Campus Bela Vista, que possibilitaram a 
execução do projeto e em especial aos professores do Departamento de Alimentos e Nutrição da Universidade Federal de Mato Grosso pelo suporte na execução do trabalho.

\section{Referências}

Abramova, I., Medrish, M., Savel'yeva, V., Romanova, A. \& Gavrilova, D. (2020). Ion chromatography in the liqueurs and spirits quality control. In E3S Web of Conferences, 210, 3001. EDP Sciences. https://doi.org/10.1051/e3sconf/202021003001

Almeida, E. L., Lima, C. L., Borges, V. T. N., Martins, R. N. \& Batalini, C. (2012). Elaboração de licor de casca de tangerina (Citrus reticulata Blanco), variedade pokan, com diferentes concentrações de casca e tempos de processamento. Alimentos e Nutrição, 23(2), 7. http://servbib.fcfar.unesp.br/seer/index.php/alimentos/article/view/1950/1950

Anjos, O., Pedro, S. I., Caramelo, D., Semedo, A., Antunes, C. A. L., Canas, S. \& Caldeira, I. (2021). Characterization of a spirit beverage produced with strawberry tree (Arbutus unedo L.) fruit and aged with oak wood at laboratorial scale. Applied Sciences, 11 (11), 5065. 10.3390/app11115065

Association of Oficial Analytical Collaboration International - AOAC (1997). Official Methods of Analysis. Arlington.

Bhuyan, D. J., Vuong, Q. van, Chalmers, A. C., Altena, I. A. van, Bowyer, M. C. \& Scarlett, C. J. (2015). Microwave-assisted extraction of Eucalyptus robusta leaf for the optimal yield of total phenolic compounds. Industrial Crops and Products, 69, 290-299. https://doi.org/10.1016/j.indcrop.2015.02.044

Brand-Williams, W., Cuvelier, M. E. \& Berset, C. (1995) Use of a free radical method to evaluate antioxidant activity. Food Science and Technology, 28, 2530. https://doi.org/10.1016/S0023-6438(95)80008-5

Ministério da Saúde. (1998). Aprova o regulamento técnico referente à informação nutricional complementar. Portaria № 27 , de 13 de janeiro de 1998 . Diário Oficial da União, Brasil.

Ministério da Saúde. (2003). Aprova o regulamento técnico sobre rotulagem nutricional de alimentos embalados. $\mathrm{RDC} \mathrm{N}^{\circ} 360 / 03$, de 23 de dezembro de 2003. Diário Oficial da União, Brasil., p. 9.

Ministério da Agricultura, Pecuária e Abastecimento. (2009). Dispõe sobre a padronização, a classificação, o registro, a inspeção, a produção e a fiscalização de bebidas. Decreto $\mathrm{N}^{\circ}$ 6871, de 04 de junho de 2009. Diário Oficial da União, Brasil.

Coelho, A. A. M., Paula, J. E. \& Espíndola, L. S. Efeito de extratos de plantas do Cerrado em dipetalogaster máxima (Uhler) (Hemiptera, Reduviidae). Revista Brasileira de Entomologia, p. 8, 2009. http://www.scielo.br/pdf/rbent/v53n3/20.pdf. 15 Jan. 2019.

Espírito Santo, B. L. S. do, Silva, É. C. da, Cândido, C. J., Silva, A. F. da, Nascimento, V. A. do, Ballard, C. R., Cazarin, C. B. B., Maróstica, M. R., Jr, Cordeiro, L. M. C., Abboud, K. Y., Carollo, C. A., Silva, D. B., Freitas, D. dos S., Ravaglia, L. M., Alcantara, G. B., Freitas, K. de C., Santos, E. F. dos, \& Hiane, P. A. (2020). Dietary fiber chemical structures and physicochemical properties of edible Pouteria glomerata fruits, native from Brazilian Pantanal. Food Research International, 137, 109576. https://doi.org/10.1016/j.foodres.2020.109576

Esquinas-Alcázar, J. (2005). Protecting crop genetic diversity for food security: political, ethical and technical challenges. Nature Reviews Genetics, v. 6, n. 12, p. 946-953. https://doi.org/10.1038/nrg1729

Franitza, L., Granvogl, M., \& Schieberle, P. (2016). Characterization of the key aroma compounds in two commercial rums by means of the sensomics approach. Journal of agricultural and food chemistry, 64(3), 637-645. https://doi.org/10.1021/acs.jafc.5b05426

Gecer, M. K., Kan, T., Gundogdu, M., Ercisli, S., Ilhan, G., \& Sagbas, H. I. (2020) Physicochemical characteristics of wild and cultivated apricots (Prunus armeniaca L.) from Aras valley in Turkey. Genet Resour Crop Evol 67, 935-945. https://doi.org/10.1007/s10722-020-00893-9

Hammer, O., Harper, O. \& Ryan, D. (2001). PAST: Paleontological Statistics package for education and data analysis. Paleontologia electronica 4(1):9pp.

Instituto Adolfo Lutz - IAL (2008). Métodos físico-químicos de análise de alimentos. 1020p.

International Life Sciences Institute do Brasil - ILSI Br. (2003). Usos e aplicações das “Dietary Reference Intakes” DRIs. São Paulo, Brasil. p. 47.

Leonarski, E., Santos, D. F., Kuasnei, M., Lenhani, G. C., Quast, L. B. \& Zanella Pinto, V. (2021). Development, chemical, and sensory characterization of liqueurs from Brazilian native fruits. Journal of Culinary Science \& Technology, 19(3), 214-227. https://doi.org/10.1080/15428052.2020.1747035

Minim, V. P. R. (2013) Análise sensorial: estudos com consumidores. (3a ed.), UFV, 332p.

Montero, L., Schmitz, O. J. \& Meckelmann, S. W. (2020). Chemical characterization of eight herbal liqueurs by means of liquid chromatography coupled with ion mobility quadrupole time-of-flight mass spectrometry. Journal of Chromatography A, 1631, 461560. https://doi.org/10.1016/j.chroma.2020.461560

Morzelle, M. C., Bachiega, P., Souza, E.C., Boas, E. V. B. V. \& Lamounier, M. L. (2015). Caracterização química e física de frutos de curriola, gariroba e murici provenientes do cerrado brasileiro. Revista Brasileira de Fruticultura, 37, 96-103. http://dx.doi.org/10.1590/0100-2945-036/14

Odello, L. \& Braceschi, G. P. (2009). Avaliação sensorial de cachaça. Química Nova, 32, 1839-1844. https://doi.org/10.1590/S0100-40422009000700029

Oliveira, E. N. A. \& Santos, D. C. (2011) Processamento e avaliação da qualidade de licor de açaí (Euterpe oleracea Mart.). Revista Instituto Adolfo Lutz, 70(4), 8. http://periodicos.ses.sp.bvs.br/pdf/rial/v70n4/v70n4a14.pdf

Oliveira, E. N. A., Santos, D. C., Santos, Y. M. G. \& Oliveira, A. A. (2014) Aproveitamento agroindustrial da graviola (Annona muricata L.) para produção de licores: Avaliação sensorial. Journal of Biotechnology and Biodiversity, v. 5, n. 1, p. 10. https://s3.amazonaws.com/academia.edu.documents/33795245/729- 
2773-1-PB.pdf?response-content-disposition=inline\%3B\%20filename\%3DJournal_of_Biotechnology_and_Biodiversit.pdf\&X-Amz-Algorithm=AWS4-HM AC-SHA256\&X-Amz-Credential=AKIAIWOWYYGZ2Y53UL3A\%2F20190625\%2Fus-east-1\%2Fs3\%2Faws4_request\&X-Amz-Date=20190625T1519 38Z\&X-Amz-Expires=3600\&X-Amz-SignedHeaders=host \&X-Amz-Signature=bca464c369391d66bc0d49a89d4d181f7e29a9c70988892f23b536697ff5d07b

Petrović, M., Pastor, F., Đurović, S., Veljović, S., Gorjanović, S., Sredojević, M. \& Vukosavljević, P. (2021). Evaluation of novel green walnut liqueur as a source of antioxidants: Multi-method approach. J Food Sci Technol 58, 2160-2169. https://doi.org/10.1007/s13197-020-04726-6

Polak, J. \& Bartoszek, M. (2015) The study of antioxidant capacity of varieties of nalewka, a traditional Polish fruit liqueur, using EPR, NMR and UV-vis spectroscopy. Journal of Food Composition and Analysis, 40, 114-119. https://doi.org/10.1016/j.jfca.2015.01.006

Roesler, R., Malta, L. G., Carrasco, L. C., Holanda, R. B., Sousa, C. A. S. \& Pastore, G. M. (2007) Atividade antioxidante de frutas do cerrado. Ciência e Tecnologia de Alimentos, 27, 53-60. https://doi.org/10.1590/S0101-20612007000100010

Schiassi, M. C. E. V., Souza, V. R., Lago, A. M. T., Campos, L. G. \& Queiroz, F. (2018) Fruits from the Brazilian Cerrado region: Physico-chemical characterization, bioactive compounds, antioxidant activities, and sensory evaluation. Food Chemistry, 245, 305-311. https://doi.org/10.1016/j.foodchem.2017.10.104

Serreli, G., Jerković, I., Gil, K. A., Marijanović, Z., Pacini, V. \& Tuberoso C. I. G. (2017) Phenolic compounds, volatiles and antioxidant capacity of white myrtle berry liqueurs. Plant Foods for Human Nutrition, 72, 205-210. https://doi.org/10.1007/s11130-017-0611-8

Sistema de Informação sobre a Biodiversidade Brasileira - SiBBR. (2019). Ficha de espécies. https://ferramentas.sibbr.gov.br/fic ha/bin/view/especie/pouteria_ramiflora

Silva, C. O., Tassi, É. M. M. \& Pascoal, G. B. (2016) Ciência dos alimentos: princípios de bromatologia. Rubio, 248p.

Silva, E., Júnior, J. R. C., Lacerda, M. \& Brandão, T. (2021). Licores de frutas: importância, riquezas e símbolos para a região nordeste do Brasil. ENCICLOPÉDIA BIOSFERA, 18(35). 10.18677/EnciBio_2021A11

Silva, J. L., Costa, F. B., Nascimento, A. M., Costa, R. T. R. V. \& Formiga, A. S. (2018) Pós-colheita de frutos de juazeiro em diferentes estádios de maturação. Revista Verde de Agroecologia e Desenvolvimento Sustentável, 13, 302. https://doi.org/10.18378/rvads.v13i3.4744

Silva, M. R., Lacerda, D. B. C. L., Santos, G. G. \& Martins, D. M. O. (2008). Caracterização química de frutos nativos do Cerrado. Ciência Rural, 38, 17901793, https://doi.org/10.1590/S0103-84782008000600051

Śliwińska, M., Wiśniewska, P., Dymerski, T., Wardencki, W. \& Namieśnik, J. (2015). The flavour of fruit spirits and fruit liqueurs: a review: The flavour of fruit spirits and fruit liqueurs. Flavour and Fragrance Journal, 30, 197-207. https://doi.org/10.1002/ffj.3237

Núcleo de Estudos e pesquisas em Alimentação - NEPA (2011). Tabela Brasileira de Composição de Alimentos - TACO. Campinas, UNICAMP. 161p.

Teixeira, L. V. (2009) Análise sensorial na indústria de alimentos. Revista Instituto de Laticínios Cândido Tostes, p. 10. https://www.revistadoilct.com.br/rilct/article/view/70/76

Teixeira, L. J. Q., Ramos, A. M., Chaves, J. B. P. \& Stringheta, P. C. (2007) Testes de aceitabilidade de licores de banana. Revista Brasileira de Agrociência, 13, 5. https://periodicos.ufpel.edu.br/ojs2/index.php/CAST/article/view/1362/1347 18

Teixeira, L. J. Q., Simões, L. S., Saraiva, S. H., Junqueira, M. S. \& Sartori, M. A. (2012) Determinação da proporção de açúcar e fruta necessários para conferir os atributos ideais a licor de abacaxi. Enciclopédia Biosfera, Centro Científico Conhecer, 14.7. http://www.conhecer.org.br/enciclop/2012a/engenharia/determinacao\%20da\%20proporcao.pdf

Valente, D. M., Sousa, J. S. \& Bastos, M. N. C. (2013) Estudo taxonômico de Sapotaceae Juss. do litoral Paraense. Acta Amazônica, 43, n161-168. https://doi.org/10.1590/S0044-59672013000200005

Venturini, W. G., Filho (2010) Bebidas Alcoólicas: Ciência e Tecnologia. Blucher, v. 1. 462p.

Villa, F., Rotili, M. C. C., Silva, D. F. D., Braga, G. C., Rosanelli, S. \& Eberling, T. (2021). Two alcoholic sources in the preparation, chemical characterization and acceptability of artisanal dovialis liquors. Rural Science, 51. https://doi.org/10.1590/0103-8478cr20200830

Woisky, R. G. \& Salatino, A. (1998) Analysis of propolis: some parameters and procedures for chemical quality control. Journal of Apicultural Research, 37, 99-105. https://doi.org/10.1080/00218839.1998.11100961

Yahia, E. M. \& Guttierrez-Orozco, F. (2011). Lucuma (Pouteria lucuma (Ruiz and Pav.) Kuntze). In Postharvest biology and technology of tropical and subtropical fruits (pp. 443-450e). Woodhead Publishing. https://doi.org/10.1533/9780857092885.443

Zhang, X., Wang, C., Wang, L., Chen, S. \& Xu, Y. (2020). Optimization and validation of a head space solid-phase microextraction-arrow gas chromatography-mass spectrometry method using central composite design for determination of aroma compounds in Chinese liquor (Baijiu). Journal of Chromatography A, 1610, 460584. https://doi.org/10.1016/j.chroma.2019.460584 